\title{
Ocean Data View 4.0
}

Murray Brown

Intergovernmental oceanographic Commission - Paris FRANCE

ABSTRACT

Ocean Data View, a multiple-variable graphical analysis and display package for oceanographic station data (station metadata, temperature, salinity, nutrients, others), compatible with several commonly used international marine data formats, is reviewed. Standard derived variables or those based on user-provided formulas can be displayed. Station charts, station data profiles, multi-variable scatter plats, section profiles, and surface plots (one variable plotted on a specific value of any other variable) can be created easily within a user-specified physical layout of Cartesian frames. Plotted data is easily gridded and/or contoured in nearly all views.

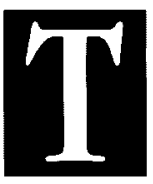

he Holy Grail of oceanware, a really good, five-cent Windows data browser, has finally appeared: Ocean Data View! When word spread this past winter that Reiner Schlitzer at the Alfred Wegener Institute-Bremerhaven had released a sensational new graphic program for marine data (http://www.awibremerhaven.de/GPH/ODV/), this reviewer wasted no time in downloading and testing it. My intention was just to see if it really worked, and - if so - could it handle some of the analysis and quality control lessons we offer in Intergovernmental Oceanographic Commission (IOC) training classes. Ocean Data View (ODV), now on version 4.0.4, turned out to be a powerful, versatile, yet easy-to-use package that instantly transforms this klutziest of users into a savvy hydrographer (well, almost). ODV is now my program of choice - its overall design, simplicity, capabilities and compatibilities set a high water mark in oceanware that will be hard to match.

I've heard about folks who are absolutely addicted to another browser, OceanAtlas (Swift, J. et al; http: / /odf.ucsd.edu/OceanAtlas/), and have seen the outstanding examples of its work in Mattias Tomczak's web-published lecture notes (http://gaea.es.flinders.edu.au/ mattom / ES1/ contents.html). But the OceanAtlas is a Macintosh application, vaguely descended from Peter Rhines' old PC ATLAST (http://podaac.jpl.nasa.gov:2031/dataset_docs/ATLA ST.html) which sprang from the Living Ocean Atlas of Stommel. Reading the literature on OceanAtlas certainly convinces me that it might be a terrific mousetrap, but alas I am a PC-head, son of a PC-head, and will never know.

Although, as I said, you never really see what's going on "inside" ODV's obviously very robust data model, the capabilities for producing graphical analyses are vast. The "data collection" (the author's term to describe all data currently combined from various sources into an internally consistent, memory-resident, integrated database) available to ODV includes the usual cruise, station, sample, and measurement fields. These can be sliced and diced in an almost limitless way, by mapping selected fields against the $X$ and $Y$ axes in a Cartesian coordinate system. The $Z$ axis (if needed) is represented by the color-coding schema for the $X-Y$ data points, resulting in plots similar to those from ATLAST. Distance along cruise tracks (or latitude, or longitude) can be an axis, resulting in section plots; time can be an axis, resulting in time-series analyses. [The ease of cutting the data into useful $X-Y$ planes (or $X-Y-Z$ spaces) from the inherent $n$-dimensional dataset reminds this reviewer of the ever-popular Grid Analysis and Display System (GrADS) meteorology data program (http://grads.iges.org/grads/), which by the way is out now in new Windows versions (!). In fact, I'd probably be recommending various kluges between ocean data and GrADS, if its data format requirements weren't so ... well ... intellectual.]

There are three very special capabilities in ODV that extend the above analytical model in powerful ways:

- The user can select from a wide suite of "canned" derived variables (including the depth derivative) to supplement the measured variables. These new fields are added instantly to the data collection. In the most recent version of the program, geostrophic velocities can be calculated from appropriate survey transects; the new values are used to populate a new "virtual" data collection tied to the original data and displayed separately.

- When the $X-Y$ plane is selected such that $X=$ longitude and $Y=$ latitude (i.e. the Cartesian graph is also a map), then the user can plot a third variable (the $Z$ variable) on any selected "surface". Surfaces are spe- 
cific values of another variable. For instance, you can plot oxygen on the 18-degree temperature surface.

- There is a macro capability, yet unexplored by this reviewer, based on a higher-order syntax defined in the on-line HELP facility.

Additional bells and whistles are also provided to enhance the visual impact of the charts. The default, low-resolution global coastline (ca. 1:20,000,000) can be replaced by "high resolution" versions (ca. 1:1,000,000) for the globe or for the Mediterranean. The old ETOPO5 bathymetry (http:// www.ngdc.noaa.gov/mgg/global/global.html) is present for bottom depiction in sections. In the graphing department, all kinds of nifty things can be done to the gridded data depictions, such as contour lines, grid coarseness control, error limits, and data-point notation. The overall menu is far too rich to cover here.

So, ODV can make all kinds of graphs, but with all this power are we locked into specific variables combinations or prescribed layouts? Are flexibility and control sacrificed in order to bring us this rich menu of possibilities? Author Schlitzer has kindly thought of everything, so the user enjoys as many display options as the underlying analytical tools. First, in any display mode, the user can instantly switch any variable along any axis; this permits rapid scanning through all sorts of multiple-property graphs with a view toward quick quality control, or water mass assessment. Second, the user can switch into a graphical layout screen that permits moving, re-sizing, adding, or deleting graphs. Third, the color-scale for the Z-variable can be expanded or contracted to suit the data better. An eerily consistent logic is at work, so that any changes to the color scale or to the range of any axis cascade through the other relevant graphs, all automatically.

\section{This all sounds very tempting, but how much trouble is it to enter the data in which I was really interested?}

Several templates for the ODV "data collection" exist, such as the variable set in the World Data Center A's new World Ocean Database 98 (WOD98; http:/ / www.nodc.noaa.gov/OC5/index.html) or the variable set in the traditional SD2 exchange format (http://www.nodc.noaa.gov/NODC-Archive/sd2.htm). Even the regrettable "mass-per-mass" WOCE data units (http://whpo.ucsd.edu/manuals.htm) are on the list, an act of supreme charity one would think, considering WOCE's apostasy in these matters. [The reviewer is an ICES true believer.] Into any template, the user can load data from several or many different recognized formats. But, be careful you don't use a template that omits an analyte of interest; for instance, don't use the SD2 template if you want to load chlorophyll data. The recognized import formats are: World Ocean Atlas 94, WOD98, SD2, WOCE, ODV's own hierarchical ASCII format, and ODV's own ASCII spreadsheet format.
There is an ingenious function that matches up the incoming variables with the template variables (based on the field names), and even performs necessary conversions when needed. The troublesome mass-per-mass variables can be handled in this way.

Data importing is aided by a well-designed set of filters to impose time, geographic, quality, source, or type limits on incoming data. This same filter is, however, always available during the graphing process, so the user can safely import the maximum amount of data initially. Veteran users of ODV who may wonder why I have not mentioned unzipping the WOD98 files will be happy to know that the current version does this automatically, which brings up a good point: Schlitzer has posted several updates to the program with little fanfare, so sign up for his mail list to receive update notices.

\section{So how good is Ocean Data View, really?}

From the very parochial perspective of the OceanPC software project (http://www.ices.dk/ocean/oceanpc.htm), where we desperately need a well-documented, Windows graphical engine to replace ATLAST, ODV offers a wide range of attractive analytical tools that are immediately grasped by students in developing countries. We can take regional data straight from WOD98, and have the students making rather sophisticated graphs in minutes. The next step, adding local data for national-and institutional-level projects, is obvious, and - what's more important - made attractive by the simplicity of the import routines. One attractive route would be raw data entry with OceanPC, then use of OceanPC's new ODV export routine. We hope eventually in the International Oceanographic Data and Information Exchange (IODE) community to reap a large fraction of these additional data, because they have already necessarily been converted to a "standard" format during the process. From this point of view, ODV is the Great Carrot, thanks to the good Professor Schlitzer.

From the broader perspective of students and professionals everywhere, ODV is definitely the best freebie around. Although there are some tricks you need to learn to get $\mathrm{CD}$ datasets into the system (for instance, there are various routes from WOD98 to ODV), the compatibility with other information models is inspiring. Author Schlitzer has incorporated important existing formats, and kept his own format-craft to a necessary minimum, a commendable restraint that we could use more of. Further, he has achieved a reputation for near-instant upgrades in response to user requests and comments. One very recent upgrade was the addition of the WOD98 format; another was explicit logging of user corrections to the data (yes, edits are possible too!). Carefully working within the context of existing widgets when they are good, but imaginatively creating new ones when they are lacking, is the crowning achievement here. That, plus the very professional 
"look and feel" of the program screens, menus, and documents. Don't miss a very good thing . . . add Ocean Data View to your software collection ASAP.

\section{SUPPLEMENTAL INFORMATION:}

How To Obtain: Web address, http://www.awi-bremerhaven.de/GPH/ODV/. Make sure to sign up for the mail list of users, because frequent updates occur without any other notice.

What to Obtain: ODV40.ZIP (2252K), ETOPO.ZIP (1416K), GLOBHR (2740K), MEDHR.ZIP (236K), WOCEBTL.ZIP (3535K).

Installation Tips: Install optional zipfiles from the recommended C: \ODV40 directory, not from C: \TEMP.

What Goes In: World Ocean Atlas 94, World Ocean Database 98, WOCE hydrographic program (bottle or CTD), SD2, and (via conversions) ICES standard profile databases; ASCII hierarchical data file; ASCII spreadsheet data file.

What Comes Out: PostScript files, GIF files, ASCII files of data points currently plotted (undocumented for- mats); ASCII file of data in currently selected stations (ODV-specific formats .03X and .04X); ASCII spreadsheet data file.

Operation Tips: The SD2 import routine in ODV may not work with SD2 files on NODC CD-ROM 20 ("Long Time Series"). Use the OceanPC software to convert SD2 files to comma-separated version (CSV); then use the special CSV-ODV program from ICES to convert CSV to ODV format. To put new data into ODV, use the OceanPC CSV spreadsheet format to create the file(s) and perform preliminary data quality control with OceanPC routines; then convert the final CSV file with the CSV-ODV program from ICES. Occasional glitches in the graphics screens can be overcome by switching the display away from and back to the desired mode.

Documentation \& Help: Excellent hyperlinked, HELPtype documentation. Export of ASCII values of currently plotted data points needs better format documentation. Other issues are dealt with quickly by direct contact with the author at the web address. 